\title{
Piezooptic coefficients and acoustooptic efficiency of TGS crystals
}

\author{
${ }^{1}$ Mytsyk B., ${ }^{2}$ Shut V., ${ }^{1}$ Demyanyshyn N., ${ }^{2}$ Mozzharov S., ${ }^{3}$ Erba A., \\ ${ }^{4}$ Kalynyak B., ${ }^{5}$ Mys O. and ${ }^{5}$ Vlokh R. \\ ${ }^{1}$ Karpenko Physico-Mechanical Institute, 5 Naukova Street, 79601 Lviv, Ukraine \\ ${ }^{2}$ Institute of Technical Acoustics of NAS of Belarus, 13 Lyudnykov Avenue, \\ 220023 Vitebsk, Belarus \\ ${ }^{3}$ Universita di Torino, Via Giuria 5, 10125 Torino, Italy \\ ${ }^{4}$ Pidstryhach Institute for the Applied Problems of Mechanics and Mathematics, \\ 3b Naukova Street, 79060, Lviv, Ukraine \\ ${ }^{5}$ Vlokh Institute of Physical Optics, 23 Dragomanov Street, 79005 Lviv, Ukraine
}

Received: 17.01 .2017

\begin{abstract}
We have determined ten piezooptic tensor components for monoclinic triglycine sulfate crystals, using an interferometric method. Acoustooptic figure of merit has been calculated for the case of isotropic acoustooptic interactions with a quasi-longitudinal acoustic wave.
\end{abstract}

Keywords: piezooptic tensor, mechanical stresses, monoclinic crystals, interferometric method.

PACS: $78.20 . \mathrm{Hp}$

UDC: 535.551

\section{Introduction}

Low-symmetry crystals manifest a number of physical properties associated with their increased anisotropy and, in particular, propose a larger variety of geometries of their efficient applications. However, the corresponding constitutive coefficients responsible for the efficiency, in particular piezooptic and acoustooptic (AO) parameters for the low-symmetry crystalline materials, are mainly represented by the tensors of ranks higher than second and, moreover, the components of these tensors are difficult for experimental studies. The most prominent examples of anisotropic material media are crystals belonging to monoclinic and triclinic systems.

The matrices of piezooptic coefficients (POCs) for all the crystals of the monoclinic point symmetry groups $2, m$ and $2 / m$ are the same and contain 20 independent POCs $\pi_{i m}$, with $i, m=1,2, \ldots, 6$. If the indices $i$ and $m$ are equal to 1,2 and 3 , the relevant POCs $\pi_{11}, \pi_{12}, \pi_{13}, \pi_{21}$, $\pi_{22}, \pi_{23}, \pi_{31}, \pi_{32}$ and $\pi_{33}$ are often called as 'principal' [1], since they describe the changes in the principal refractive indices $n_{1}, n_{2}$ and $n_{3}$ of a crystal under the action of mechanical stress tensor components $\sigma_{m}(m=1,2,3)$. The POCs with the indices $i=4,5,6$ are responsible for piezooptically induced rotations of optical indicatrix under the action of stress components $\sigma_{m}$ with $m=$ $1,2, \ldots, 6[1,2]$. These POCs are the most difficult to determine experimentally, since the theoretical relations necessary for calculations contain complicated combinations of both the POCs and the elastic compliances [1-3]. The latter fact leads to large, sometimes non-acceptable, final errors in determination of the POCs. This is a reason for the lack of data concerned with the POCs of monoclinic crystals, in spite of numerous photoelasticity data available for the crystals with higher symmetry (see, e.g., Refs. [2-5]). In the present work we will deal with the piezooptic studies of monoclinic crystals, using canonical triglycine sulfate (TGS) crystals as an example. 
TGS is characterized by the chemical formula $\left(\mathrm{NH}_{2} \mathrm{CH}_{2} \mathrm{COOH}\right)_{3} \times \mathrm{H}_{2} \mathrm{SO}_{4}$. It refers to the point symmetry group 2 at the temperatures below a ferroelectric phase transition $\left(T_{C}=49.2^{\circ} \mathrm{C}\right)$ and the group $2 / m$ above the $T_{C}$ point [6]. The TGS crystals represent a known ferroelectric material, which is well studied in many respects. In particular, it is known that it is transparent down to $240 \mathrm{~nm}$ in the ultraviolet region [7]. Notice that this feature has been predominant in our choice of TGS for the present piezooptic studies. It is worthwhile that the piezooptic effect in TGS has been studied in a number of works (see, e.g., Refs. [8, 9]). However, in the most of cases only some POC combinations have been determined using the measurements of optical retardation, instead of the individual POCs themselves. In addition, the methods used so far for this aim have not been completely correct. For instance, the errors caused by wedged samples (see the analysis [10]) and inhomogeneous distribution of mechanical stress components inside uniaxially loaded samples (see Ref. [11]) have not been accounted for in the literature, thus making the final results inaccurate. Elastooptic coefficients of the TGS crystals have been measured in Ref. [12] with a Dixon-Cohen method, so that the signs of these coefficients are still unknown. Notice that those signs are needed while analyzing anisotropy of AO figure of merit that governs the AO efficiency of material (see, e.g., Ref. [13]). It can be obtained after recalculating the POCs into elastooptic coefficients.

The aim of the present work is to determine the principal POCs for the low-symmetry TGS crystals.

\section{Experimental techniques}

The absolute values of POCs for the TGS crystals were determined using a known interferometric method. The experimental equipment was built basing on a single-path Mach-Zehnder interferometer, in one of the arms of which a sample was introduced. The change in the optical path $\delta \Delta_{k}$ in this arm of interferometer caused by the uniaxial pressure described by the stress tensor component $\sigma_{m}$ is given by the relation [1]

$$
\delta \Delta_{k}=-\frac{1}{2} \pi_{i m} \sigma_{m} d_{k} n_{i}^{3}+S_{k m} \sigma_{m} d_{k}\left(n_{i}-1\right) .
$$

Here $n_{i}$ denotes the refractive index of a crystal under test, $d_{k}$ the sample thickness along the light beam, and $S_{k m}$ are the elastic compliance coefficients. The indices $k, i$ and $m$ denote respectively the directions of the light beam, its polarization and the uniaxial pressure. If $\delta \Delta_{k}$ in Eq. (1) is substituted with $\lambda / 2$, where $\lambda=632.8 \mathrm{~nm}$ is the light wavelength used in this work, the parameter $\sigma_{m}=\sigma_{i m}$ is referred to as a half-wave stress. The relations for the POC $\pi_{i m}$ expressed in terms of the half-wave (or control) stresses can be written as

$$
\pi_{i m}=-\frac{\lambda}{n_{i}^{3} d_{k} \sigma_{i m}}+\frac{2 S_{k m}}{n_{i}^{3}}\left(n_{i}-1\right)=-\frac{\lambda}{n_{i}^{3} \sigma_{i m}^{\mathrm{o}}}+\frac{2 S_{k m}}{n_{i}^{3}}\left(n_{i}-1\right) .
$$

In eq. (2), $\sigma_{i m}^{o}=d_{k} \sigma_{i m}$ is the control mechanical stress, which is a characteristic of material, and the half-wave stress $\sigma_{i m}$ characterizes rather a sample, since it depends on its size.

Notice that even apparently plane-parallel samples manifest a small lateral wedging. In practice, then the maximum and minimum thicknesses of the sample along the light beam direction differ by $\sim 1-5 \mu \mathrm{m}$, when the sample height and the mean thickness are equal to $\sim 8 \mathrm{~mm}$. This wedging effect can be considered when substituting $1 / \sigma_{i m}^{o}$ in Eq. (2) with the mean of the appropriate inverse control stresses $\sigma_{i m}^{*}$ and $\sigma_{i m}^{\prime *}$, such that we obtain 


$$
\frac{1}{\sigma_{i m}^{\mathrm{o}}}=\frac{1}{2}\left(\frac{1}{\sigma_{i m}^{*}}+\frac{1}{\sigma_{i m}^{\prime *}}\right) .
$$

Here $\sigma_{i m}^{*}$ and $\sigma_{i m}^{\prime *}$ are the control mechanical stresses determined for the sample placed in some initial position and the sample rotated by $180^{\circ}$ around the light beam, respectively. We refer the reader to our recent works $[10,14,15]$ for more detailed explanations of the effect of sample wedging on the POCs measurements. Notice that our samples indeed possessed a small wedging (sometimes it was as large as $3.5 \times 10^{-2} \mathrm{deg}$ ). On the other hand, the vertical displacement of the samples under the mechanical stresses $\sigma_{m}$ was negligibly small $(\sim 0.01 \mathrm{~mm})$, owing to high rigidity of a sample holder. Therefore, the control stresses $\sigma_{i m}^{o}, \sigma_{i m}^{*}$ and $\sigma_{i m}^{\prime *}$ in our experiments with the TGS crystals were the same.

As mentioned in Ref. [11], the actual mechanical stress appearing in the central part of a sample is usually larger when compared with the stress applied. On the basis of many experiments we have found that, for the samples of cubic shape $\left(8 \times 8 \times 8 \mathrm{~mm}^{3}\right)$ used by us, the control stress in the central part of the sample is $10 \%$ higher than the stress applied.

Our single crystals were grown from aqueous solution at the temperature $34.5^{\circ} \mathrm{C}$ somewhat lower than the point of solution saturation $\left(35.8^{\circ} \mathrm{C}\right)$. The measurements of POCs were carried out in the coordinate system $X_{1} X_{2} X_{3}$ of Fresnel ellipsoid, which is rotated with respect to the Cartesian system $X Y Z$ by the angle $3^{\circ}$ around the two-fold symmetry axis. As seen from Fig. 1, the latter coincides with the crystallographic axis $b$ and the axis $X_{2}[16,17]$. In fact, the crystallographic system is not rectangular for the monoclinic symmetry groups and the monoclinic angle in the organic TGS crystals is large enough, $\sim 105$ deg [17]. In such a case, the axes defined crystallographically are the axis $b\left(Y\right.$ or $\left.X_{2}\right)$ and the axis $c(Z)$.

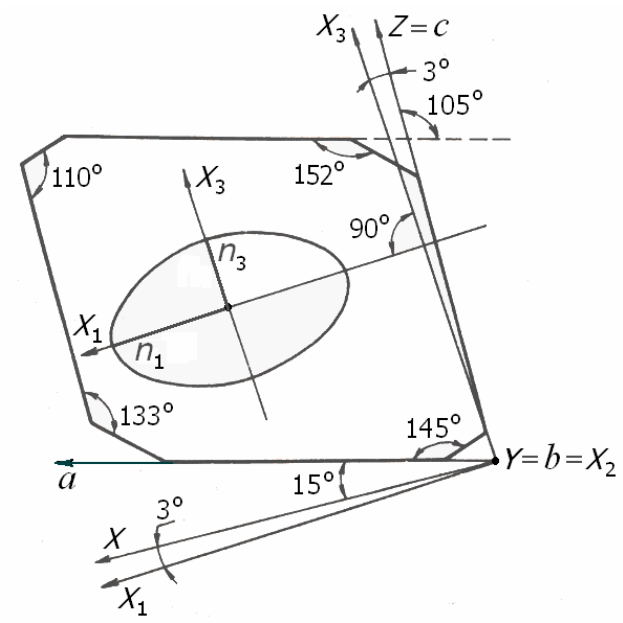

Fig. 1. Habitus of TGS crystals and coordinate systems: a crystallographic system (abc), a Cartesian one $(X Y Z)$, and a system based on the eigenvectors of Fresnel ellipsoid $\left(X_{1} X_{2} X_{3}\right)$.

We remind that the rectangular coordinate system $X Y Z$ is usually used when determining such physical quantities as the elastic stiffness and compliance coefficients, or the piezoelectric coefficients [16]. Following from the peculiarities of polarimetric-interferometric experiments performed in the present work, it would be reasonable to use the coordinate system $X_{1} X_{2} X_{3}$ of the Fresnel ellipsoid. Therefore the elastic compliance coefficients $S^{\prime}{ }_{k m}$ obtained by us from the elastic stiffness coefficients experimentally determined in Ref. [16] for the coordinate system $X Y Z$ 
$\left(S_{12}^{\prime}=-2.9 \times 10^{-12}, S_{13}^{\prime}=-22.7 \times 10^{-12}\right.$ and $\left.S_{23}^{\prime}=-57.7 \times 10^{-12} \mathrm{~m}^{2} / \mathrm{N}\right)$ have to be recalculated for the coordinate system $X_{1} X_{2} X_{3}$, using the full matrix of elastic compliances. The respective coefficients in the coordinate system $X_{1} X_{2} X_{3}$ are as follows: $S_{12}=-0.49 \times 10^{-12}, S_{13}=-27.7 \times 10^{-12}$ and $S_{23}=-60.1 \times 10^{-12} \mathrm{~m}^{2} / \mathrm{N}$. The $S_{k m}$ values thus obtained are then used for calculating the POCs $\pi_{i m}$.

Under normal conditions, the principal refractive indices of TGS crystals are equal to $n_{1}=1.591, n_{2}=1.488$ and $n_{3}=1.563$ at $\lambda=632.8 \mathrm{~nm}$ [18]. Notice that further calculations of the elastooptic coefficient $p_{13}$ need the $\pi_{15}$ component. It can be determined using a so-called ' $X_{2} / 45^{\circ}$ sample' cut, as explained in Ref. [1].

\section{Results and discussion}

The control stresses $\sigma_{i m}^{o}$ determined in our experiments and the corresponding principal POCs calculated with Eq. (2) are presented in Table 1. The $\sigma_{i m}^{\mathrm{o}}$ and $\pi_{i m}$ values $(i, m=1,2,3)$ have been obtained using the samples of so-called 'direct cuts', of which faces are perpendicular to the axes $X_{1}, X_{2}$ or $X_{3}$. For example, Eq. (2) under the experimental conditions described by the line 1 in Table $1(i=m=1, k=2)$ reads as

$$
\pi_{11}=-\frac{\lambda}{n_{1}^{3} \sigma_{11}^{o}}+\frac{2 S_{12}}{n_{1}^{3}}\left(n_{1}-1\right) .
$$

Theoretical relations for the other experimental conditions can be obtained in a similar manner.

Table 1. Control stresses $\sigma_{i m}^{\mathrm{o}}$ and absolute POCs $\pi_{i m}$ determined for the TGS crystals.

\begin{tabular}{|c|c|c|c|c|c|}
\hline \multirow{2}{*}{$\begin{array}{l}\text { Number of } \\
\text { experiment }\end{array}$} & \multicolumn{3}{|c|}{ Experimental conditions } & \multirow{2}{*}{$\sigma_{i m}^{\mathrm{o}}, \mathrm{kg} / \mathrm{cm} *$} & \multirow{2}{*}{$\begin{array}{c}\pi_{i m}, \mathrm{Br} \\
\left(1 \mathrm{Br}=10^{-12} \mathrm{~m}^{2} / \mathrm{N}\right)\end{array}$} \\
\hline & $m$ & $k$ & $i$ & & \\
\hline 1 & 1 & 2 & 1 & $\sigma_{11}^{\mathrm{o}}=-275$ & $\pi_{11}=-0.73 \pm 0.06$ \\
\hline 2 & 1 & 2 & 3 & $\sigma_{31}^{\mathrm{o}} \Rightarrow \infty$ & $\pi_{31}=-0.14 \pm 0.01$ \\
\hline 3 & 1 & 3 & 1 & $\sigma_{11}^{\mathrm{o}}=20.5$ & $\pi_{11}=-0.31 \pm 0.91$ \\
\hline 4 & 1 & 3 & 2 & $\sigma_{21}^{o}=22.0$ & $\pi_{21}=0.7 \pm 1.0$ \\
\hline 5 & 2 & 1 & 2 & $\sigma_{22}^{\mathrm{o}}=35.5$ & $\pi_{22}=5.40 \pm 0.55$ \\
\hline 6 & 2 & 1 & 3 & $\sigma_{32}^{o}=280$ & $\pi_{32}=0.46 \pm 0.06$ \\
\hline 7 & 2 & 3 & 1 & $\sigma_{12}^{o}=9.7$ & $\pi_{12}=-1.1 \pm 1.9$ \\
\hline 8 & 2 & 3 & 2 & $\sigma_{22}^{\mathrm{o}}=8.5$ & $\pi_{22}=5.25 \pm 2.50$ \\
\hline 9 & 3 & 1 & 2 & $\sigma_{23}^{\mathrm{o}}=15.0$ & $\pi_{23}=4.9 \pm 1.4$ \\
\hline 10 & 3 & 1 & 3 & $\sigma_{33}^{\mathrm{o}}=11.5$ & $\pi_{33}=6.5 \pm 1.5$ \\
\hline 11 & 3 & 2 & 1 & $\sigma_{13}^{\mathrm{o}}=6.2$ & $\pi_{13}=8.2 \pm 2.7$ \\
\hline 12 & 3 & 2 & 3 & $\sigma_{33}^{\mathrm{o}}=7.2$ & $\pi_{33}=5.8 \pm 2.5$ \\
\hline
\end{tabular}

* The signs $+/-$ of the $\sigma_{i m}^{o}$ parameter imply that the optical path in the interferometer arm containing the sample increases/decreases under the mechanical pressure. 
Note that the coefficient $\pi_{11}$ can also be determined under the experimental conditions $i=m=1$ and $k=3$ (see the line 3 in Table 1). However, the error in this case $\left(\delta \pi_{11}= \pm 0.91 \mathrm{Br}\right)$ is notably larger than that typical for the experimental geometry mentioned above $( \pm 0.06 \mathrm{Br})$. Thus, we accept for further calculations the $\pi_{11}$ value determined for the first type of experiment (the experiment \#1 in Table 1). The coefficients $\pi_{22}$ and $\pi_{33}$ have also been determined in the two experimental geometries (see the lines 5, 8 and 10,12, respectively). Under the conditions described by the lines 5 and 10 in Table 1, the errors are smaller than those peculiar for the conditions described by the lines 8 and 12. Therefore the corresponding POC value should be accepted as more accurate.

Let us notice that the coefficients $\pi_{12}$ and $\pi_{21}$ are close to zero (see the lines 4 and 7 in Table 1). At the same time, the data of Table 1 testifies that the coefficients $\pi_{13}, \pi_{22}$ and $\pi_{33}$ are relatively large. These POCs are higher than those measured for such well-known $\mathrm{AO}$ materials as $\mathrm{GaP}$ (the maximal POC being equal to $1.44 \mathrm{Br}[15]), \mathrm{LiNbO}_{3}(\sim 2.1 \mathrm{Br}[14,19]), \beta-\mathrm{BaB}_{2} \mathrm{O}_{4}$ (3.7 $\mathrm{Br}[20])$ and crystalline quartz $(3.11 \mathrm{Br}[21])$.

Now we consider the piezooptic efficiency of TGS, which determines practical possibilities for its applications for photoelastic light modulation cells and photoelastic pressure sensors $[14,15]$. This parameter can be defined as $\delta \Delta_{k} /\left(d_{k} \sigma_{m}\right)$, i.e. as the optical path change $\delta \Delta_{k}$ per unit sample thickness $d_{k}$ along the direction of light propagation and per unit mechanical stress $\sigma_{m}$ [3]. The relation for the piezooptic efficiency is given by

$$
\frac{\delta \Delta_{k}}{d_{k} \sigma_{m}}=-\frac{1}{2} \pi_{i m} n_{i}^{3}+S_{k m}\left(n_{i}-1\right)
$$

As an example, we obtain $\delta \Delta_{2} /\left(d_{2} \sigma_{3}\right)=-52.0 \mathrm{Br}$ for the geometry at which the highest POC value is achieved ( $i=1, m=3$ and $k=2$ - see the line 11 in Table 1). Notice that the piezooptic efficiency of TGS is higher than those for, e. g., lithium niobate $(-13.7 \mathrm{Br}$ [14]) or gallium phosphide (+20.3 $\mathrm{Br}[15])$. High piezooptic efficiencies associated with large POCs $\pi_{33}$ and $\pi_{22}$ and large compliance coefficient $S_{23}$ are also typical for the experimental geometries described by the lines 12 and 8 in Table $1\left(-44.9\right.$ and $-38.0 \mathrm{Br}$ ). Since the $\pi_{12}$ and $\pi_{21}$ coefficients are close to zero (see the lines 4 and 7 in Table 1), the specific changes in the optical path originating from those coefficients $(-14.7$ and $-33.3 \mathrm{Br}$, respectively) are caused only by the elastic contribution in Eq. (1), i.e. by the Poisson strain. This kind of the piezooptic effect can be conventionally called as 'imaginary'.

Finally, we wish to estimate the AO figure of merit for TGS for the isotropic AO interaction case, when the incident optical wave polarized parallel to the $X_{1}$ axis interacts with the quasilongitudinal acoustic wave propagating along the $X_{3}$ axis. Then the relation for the AO figure of merit reads as

$$
M_{2}=\frac{n_{1}^{6} p_{e f}^{3}}{\left(\rho v_{33}^{3}\right)},
$$

where $p_{\mathrm{ef}}=p_{13}$ is the effective elastooptic coefficient, $\rho=1680 \mathrm{~kg} / \mathrm{m}^{3}$ [17] the crystal density, $v_{33}=3969 \mathrm{~m} / \mathrm{s}$ the velocity of the acoustic wave propagating along the axis $X_{3}$. The latter has been calculated using the Cristoffel equation and the elastic stiffness coefficients obtained in Ref. [16] for the coordinate system $X Y Z$. The tensor component $p_{13}$ is calculated as

$$
p_{13}=\pi_{11} C_{13}+\pi_{12} C_{23}+\pi_{13} C_{33}+\pi_{15} C_{35} .
$$


Here the stiffness coefficients $C_{13}=19.9, C_{23}=20.7, C_{33}=25.3$ and $C_{35}=-4.1 \mathrm{GPa}$ have been recalculated for the coordinate system $X_{1} X_{2} X_{3}$, basing on the elastic stiffness matrix [16], while the parameter $\pi_{15}$ is equal to $-6.3 \mathrm{Br}$. The effective elastooptic coefficient thus determined amounts to $p_{13}=+0.196$. It agrees well with the value $|0.175|$ obtained with the Dixon-Cohen method [12].

In order to check further our results, we have computed this coefficient by using a quantummechanical approach recently implemented into the CRYSTAL program [22-24]. A hybrid functional augmented by a correction to take into account weak dispersive interactions (B3LYPD3) has been used within the density-functional-theory (DFT). The structure was fully-relaxed: $a=9.3845, b=12.6108, c=5.5317 \AA$, and $\beta=110.21^{\circ}$ is the angle between the directions (101) and (100). The result, $p_{13}=+0.250$, differs only $20 \%$ from our experimental data. Moreover, the positive sign of this coefficient is also confirmed by the theory.

Now we consider the $\mathrm{AO}$ interaction at which the coefficient $p_{13}$ plays a role of the effective elastooptic coefficient. As seen from Fig. 2, there are two kinds of the isotropic AO interactions that involve this effective coefficient: (a) the Bragg diffraction in the plane $X_{2} X_{3}$ and (b) the collinear diffraction of a 'reflection' type occurring in the plane $X_{1} X_{3}$.
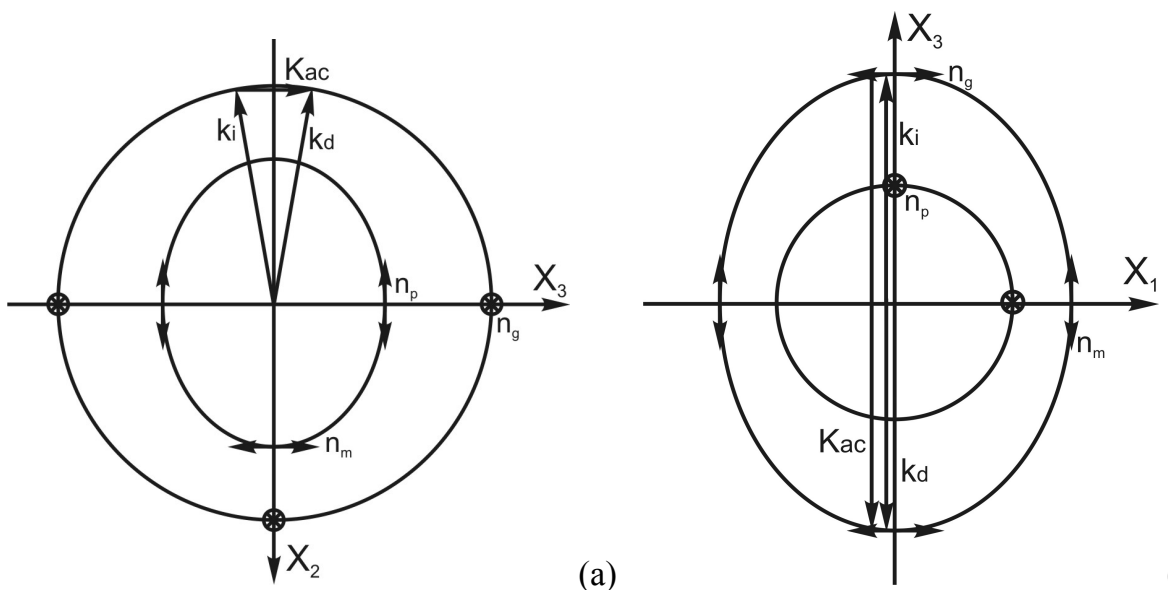

(b)

Fig. 2. Schematic vector diagrams of AO diffraction in TGS crystals with $p_{e f}=p_{13}$ : (a) Bragg diffraction in the $X_{2} X_{3}$ plane and (b) collinear diffraction of reflection type in the $X_{1} X_{3}$ plane. Double-side arrows and crossed circles indicate polarizations of the interacting optical waves; $k_{i}, k_{d}$ and $K_{a c}$ denote respectively the wave vectors of the incident and diffracted optical wave and the acoustical wave.

For the both diffraction schemes, the AO figure of merit has the same value, $M_{2}=5.9 \times 10^{-15} \mathrm{~s}^{3} / \mathrm{kg}$. This value is much smaller than that typical for the best $\mathrm{AO}$ materials such as paratellurite [25], $\mathrm{HgCl}_{2}$ [26] and $\mathrm{Sn}_{2} \mathrm{P}_{2} \mathrm{~S}_{6}$ [27] crystals, or some chalcogenide and ferroelastic crystals (see Refs. [28, 29]). However, the above AO figure of merit is still comparable or even higher than the corresponding parameters of the other crystals transparent in the ultraviolet spectral region, e.g. quartz, $\mathrm{KDP}, \mathrm{ADP}$ or $\mathrm{Li}_{2} \mathrm{~B}_{4} \mathrm{O}_{7}[21,30,31]$. It is important that our consideration has dealt with the $\mathrm{AO}$ interaction with the fast quasi-longitudinal acoustic wave. It is obvious that the interaction with slower quasi-transverse waves should lead to increasing AO figure of merit [31]. Moreover, since the TGS is a low-symmetry crystal, one can expect a large variety of geometries of AO interactions, among which there can be the geometries with higher elastooptic effect [32-34] and so with higher AO figures of merit. Such an analysis must be based on the information on all the 
components of piezooptic and elastooptic tensors. It will be carried out in our forthcoming article. Finally, we have to notice that the coordinate systems $X_{1} X_{2} X_{3}$ and $X Y Z$ differ only slightly from each other. Thus, within the small error limits, the piezooptic coefficients obtained by us for the coordinate systems $X_{1} X_{2} X_{3}$ and $X Y Z$ are the same.

\section{Conclusions}

In this work we have determined experimentally the ten components of the piezooptic matrix for TGS, the nine principal components and the component $\pi_{15}$. We have found that the component $\pi_{13}$ is the highest, $\pi_{13}=8.2 \mathrm{Br}$. An untypically large change in the optical path $(-52.0 \mathrm{Br})$ has been detected for one of the piezooptic interaction geometries. In some of experimental geometries, the mechanical loading leads to a so-called 'imaginary' piezooptic effect, at which almost all of the total optical path increment appears due to the change in the sample length but not due to the piezooptic change in the refractive indices themselves. The AO figure of merit calculated for the case of isotropic interaction with the quasi-longitudinal acoustic wave is equal to $5.9 \times 10^{-15} \mathrm{~s}^{3} / \mathrm{kg}$.

\section{Acknowledgement}

The authors acknowledge financial support of the present study from the Ministry of Education and Science of Ukraine, State Fund for Fundamental Researches of Ukraine (Project F73/1012016) and Belarusian Republican Foundation for Fundamental Research (Project F16K-022).

\section{References}

1. Mytsyk B, 2003. Methods for the studies of the piezo-optical effect in crystals and the analysis of experimental data. I. Methodology for the studies of piezo-optical effect. Ukr. J. Phys. Opt. 4: 1-26.

2. Narasimhamurty T S, Photo-elastic and electro-optic properties of crystals. N Y: Plenum Press, 1981.

3. Mytsyk B G, Photoelasticity of anisotropic materials. Lviv: Liga-Press, 2012.

4. Gorbach S S, Pakhnev A V and Shaskolskaya M P, 1974. Photoelastic properties of crystals. Rev. Electron. Tech., Ser. Mater. 16(256): 1-86.

5. Feldman A, Horowitz D, Waxler R M and Dodge M J, Optical materials characterization. National Bur. Stand. (USA): Tech. Note 993, 1979.

6. Zheludev I S, Physics of crystalline dielectrics. Moscow: Nauka, 1968.

7. Hilczer B and Balanicka S, 1973. Influence of defects on the optical behaviour of TGS crystals near the fundamental absorption edge. Phys. stat. sol. (a). 19: 717-723.

8. Romanyuk N A, Mytsyk B G and Kulyk L N, 1986. Piezo-change of the optical properties of triglycine sulfate crystals. Ukr. Fiz. Zhurn. 31: 354-359.

9. Mytsyk B G and Romanyuk N A, 1983. The nature of temperature anomalies of the piezooptical coefficient in crystals of the triglycine sulfate group. Ukr. Fiz. Zhurn. 28: 538542.

10. Mytsyk B, Demyanyshyn N, Martynyuk-Lototska I and Vlokh R, 2011. Piezo-optic, photoelastic and acousto-optic properties of $\mathrm{SrB}_{4} \mathrm{O}_{7}$ crystals. Appl. Opt. 50: 3889-3895.

11. Vasylkiv $\mathrm{Yu}$, Kvasnyuk O, Krupych O, Mys O, Maksymuk $\mathrm{O}$ and Vlokh R, 2009. Reconstruction of 3D stress fields basing on piezooptic experiment, Ukr. J. Phys. Opt. 10: $22-37$.

12. Strukov B A, Davtyan A V, Minaeva K A and Gornayev A A, 1983. Acoustooptic properties of TGS crystals. Izv. AN SSSR, Ser. Fiz. 47: 611-615. 
13. Mys O, Krupych O and Vlokh R, 2016. Anisotropy of an acousto-optic figure of merit for $\mathrm{NaBi}\left(\mathrm{MoO}_{4}\right)_{2}$ crystals. Appl. Opt. 55: 7941-7955.

14. Mytsyk B G, Andrushchak A S, Demyanyshyn N M, Kost' Y P, Kityk A V, Mandracci P and Schranz W, 2009. Piezo-optic coefficients of MgO-doped $\mathrm{LiNbO}_{3}$ crystals. Appl. Opt. 48: 1904-1911.

15. Mytsyk B G, Andrushchak A S and Kost' Y P, 2012. Static photoelasticity of gallium phosphide crystals. Crystallogr. Rep. 57: 124-130.

16. Konstantinova V P, Silvestrova I M and Aleksandrov K S, 1959. Obtaining of triglycine sulphate crystals and their physical properties. Kristallogr. 4: 69-73.

17. Wood E A and Holden A N, 1957. Monoclinic glycine sulphate: crystallographic data. Acta Cryst. 10: $145-146$.

18. Romanyuk M O, Kostetskyi O M and Viblyi I F, 1976. Dispersion and temperature dependence of refractive indices of pure triglycine sulphate crystals. Ukr. Fiz. Zhurn. 21: 207-209.

19. Krupych O, Savaryn 1983 and Vlokh R, 2014. Precise determination of full matrix of piezooptic coefficients with a four-point bending technique: the example of lithium niobate crystals. Appl. Opt. 53: B1-B7.

20. Andrushchak A S, Adamiv V T, Krupych O M, Martynyuk-Lototska I Yu, Burak Ya V and Vlokh R O, 2000. Anisotropy of piezo-and elastooptical effect in $\beta-\mathrm{BaB}_{2} \mathrm{O}_{4}$ crystals. Ferroelectrics. 238: 299-305.

21. Shaskolskaya M P. Acoustic crystals. Moscow: Nauka, 1982.

22. Erba A and Dovesi R, 2013. Photoelasticity of crystals from theoretical simulations. Phys. Rev. B. 88: 045121/1-8.

23. Erba A, Ruggiero M T, Korter T M and Dovesi R, 2015. Piezo-optic tensor of crystals from quantum-mechanical calculations. J. Chem. Phys. 143: 144504/1-8.

24. Mytsyk B, Erba A, Demyanyshyn N and Sakharuk O, 2016. Piezo-optic and elasto-optic effects in lead molibdate crystals. Opt. Mater. 62: 632-638.

25. Yano $\mathrm{T}$ and Watanabe A, 1974. Acousto-optic figure of merit of $\mathrm{TeO}_{2}$ for circularly polarized light. J. Appl. Phys. 45: 1243-1245.

26. Singh N B and Duval W M B, 1991. Growth kinetics of physical vapour transport processes: Crystal growth of opto-electronic material mercurous chloride. NASA Technical Memorandum. 103788.

27. Martynyuk-Lototska I Yu, Mys O G, Grabar A A, Stoika I M, Vysochanskii Yu M and Vlokh R O, 2008. Highly efficient acousto-optic diffraction in $\mathrm{Sn}_{2} \mathrm{P}_{2} \mathrm{~S}_{6}$ crystals. Appl. Opt. 47: 52-55.

28. Gottlieb M, Isaacs T J, Feichtner J D and Roland G W, 1974. Acousto-optic properties of some chalcogenide crystals. J. Appl. Phys. 45: 5145-5151.

29. Vlokh R and Martynyuk-Lototska I, 2009. Ferroelastic crystals as effective acoustooptic materials. Ukr. J. Phys. Opt. 10: 89-99.

30. Krupych O, Mys O, Kryvyy T, Adamiv V, Burak Y and Vlokh R, 2017. Photoelastic properties of lithium tetraborate crystals. Appl. Opt. 56: 10457-10462.

31. Mys O, Kryvyy T, Krupych O and Vlokh R, 2017. Anisotropy of acoustooptic figure of merit for lithium tetraborate crystals. J. Mod. Opt. (to be published).

32. Demyanyshyn N M, Mytsyk B G and Sakharuk O M, 2014. Elasto-optic effect anisotropy in strontium borate crystals. Appl. Opt. 53: 1620-1628. 
33. Mytsyk B G and Demyanyshyn N M, 2006. Piezo-optic surfaces of lithium niobate crystals. Crystallogr. Rep. 51: 653-660.

34. Demyanyshyn N M, Mytsyk B G, Kost' Ya P, Solskii I M and Sakharuk O M, 2015. Elastooptic effect anisotropy in calcium tungstate crystals. Appl. Opt. 54: 2347-2355.

Mytsyk B., Shut V., Demyanyshyn N., Mozzharov S., Erba A., Kalynyak B., Mys O. and Vlokh R. 2017. Piezooptic coefficients and acoustooptic efficiency of TGS crystals. Ukr.J.Phys.Opt. 18: 46 - 54.

Анотація. Інтерферометричним методом експериментально визначено десять компонент n’єзооптичного тензора для кристалів тригліцинсульфату. Розраховано коефіцієнт акустооптичної якості ичих кристалів для ізотропної акустооптичної взаємодії з квазіпоздовжною акустичною хвилею. 